\title{
THE GENETICAL CONSEQUENCES OF ASSORTATIVE MATING
}

\author{
E. LESLIE BREESE \\ Agricultural Research Council Unit of Biometrical Genetics, \\ Department of Genetics, University of Birmingham
}

\section{INTRODUCTION}

Received 27.i.56

THE disposition of the heritable portion of continuous variation under different systems of mating is of considerable interest both to the geneticist and to the plant or animal breeder. For many of the more usual experimental mating systems the behaviour of such variability can be predicted with some precision (e.g. selfing, random and sibmating). Assortative (i.e. like to like) mating has, however, in the past received less attention. Wright (I92I) has pointed out that, provided the number of genes involved is large, such a system is unlikely to achieve a greater genetic correlation (i.e. correlation between allelic genes) than is obtained under random mating. Hence, initially little increase in homozygosity would be achieved compared with a system of random mating. But at the same time we should expect an increased association between parents for genes having the same overall potential, an increase in what may be termed the genotypic correlation, and it is in the consequences of such a correlation that our present interests lie.

Experiments were devised therefore to investigate within a common parental population changes in heritable variation effected by assortative mating $(A)$ as compared with selfing $(F)$ and random mating $(R)$ systems. Mather (1949a and $b$ ) has described how the heritable portion of continuous variation may be partitioned into various components and the way in which these may be apportioned to fit certain experimental populations. These methods have been used to describe and assess changes in heritable variation during the course of inbreeding experiments (Bateman and Mather, I95I ; Mather and Vines, 1952; Hayman, 1953), and they can be extended to deal with assortative mating and so serve as a basis for comparing the genetical consequences of the three mating systems under consideration.

\section{DISTRIBUTION OF VARIABILITY}

In a randomly breeding population the variation exhibited by any continuously varying character may be partitioned into three components, provided that genic interaction be neglected. These are $\mathrm{D}$, measuring the fixable component, $\mathrm{H}$, depending on the departure of the heterozygote from the mean of the two corresponding homozygotes, and $\mathrm{E}$, measuring non-heritable variation. Where each gene is represented by two allelomorphs having equal frequencies, the 
variance of the individuals within such a population may then be expressed as $\frac{1}{2} \mathrm{D}+\frac{1}{4} \mathrm{H}+\mathrm{E}$ (see Mather, r $949 a$ and $b$ ).

The three different types of progeny which can be obtained from a randomly breeding population by imposing the mating systems with which we are concerned, each yield three second degree statistics. These are (I) the variance of family means (Ist rank variances), (2) the mean variance within families (2nd rank variances), and (3) the covariance of family means and parental mean values. Following the biometrical method described by Mather, the composition of the various statistics may be derived in terms of $\mathrm{D}, \mathrm{H}$ and $\mathrm{E}$. In the case of $F$ and $R$ generations the expectations have already been formulated and are reproduced for purposes of comparison in table I. The derivation of corresponding expectations for the $A$ generation, however, involves further basic premises to which we must now turn.

Consider two genes $A-a$ and $B-b$ where the capital letter denotes the allele giving greater expression of the character in question. Assuming the absence of dominance and interaction, and that genes are equal in effect (i.e. $d a=d b ; h a=h b=0$ ), the nine possible genotypes in a randomly breeding population can be assorted phenotypically into five intramating groups provided that environmental fluctuations are negligible. These are (1) $A A B B$, (2) $A A B b ; A a B B$, (3) $A A b b$; $A a B b$; $a a B B$, (4) $a a B b$; $A a b b$, and (5) $a a b b$, and will occur in the ratio I $: 4: 6: 4:$ I respectively. Letting $d_{a}$ and $d_{b}$ measure the departure of $A A$ and $B B$; and $--d_{a}$ and $-d_{b}$ the departure of $a a$ and $b b$ from the mid-parent, the variance of means of families derived from these mating groups will be

$$
\frac{1}{4}\left(d_{a}^{2}+d_{b}^{2}\right)+\frac{1}{8}\left(d_{a}+d_{b}\right)^{2}
$$

When $n$ genes of equal effect are considered, this term may be expressed as

$$
\frac{1}{4} \Sigma d_{a}^{2}+\frac{\mathrm{I}}{4^{n}}\left(\Sigma d_{a}\right)^{2}=\frac{1}{2} \mathrm{G}
$$

where $\mathrm{G}$ denotes the prospective genetic variation in the inbred (homozygous) population assuming equal frequencies and independent assortment of the genes.

By comparison, the variance of $R$ family means is $\frac{1}{4} \Sigma d_{a}^{2}\left(=\frac{1}{4} \mathrm{G}\right)$, so that assortatively mating the parents increases the genetic variation between families by $\frac{\mathrm{I}}{4^{n}}\left(\Sigma d_{a}\right)^{2}$, provided that genotypic correlation is complete. Expansion of this term indicates that only $\frac{\mathrm{I}}{4^{n}}$ of this increased variance is contributed by the independent deviations of homozygotes from the operative mean (expressed as $\Sigma d_{a}^{2}$ and corresponding to Mather's D) the remaining $\frac{n-\mathrm{I}}{4^{n}}$ being dependent upon product terms $d_{a} d_{b}$, etc. deriving from an excess of reinforcing homozygotic/homozygotic gene combinations $(A A B B$ and $a a b b$, where two 


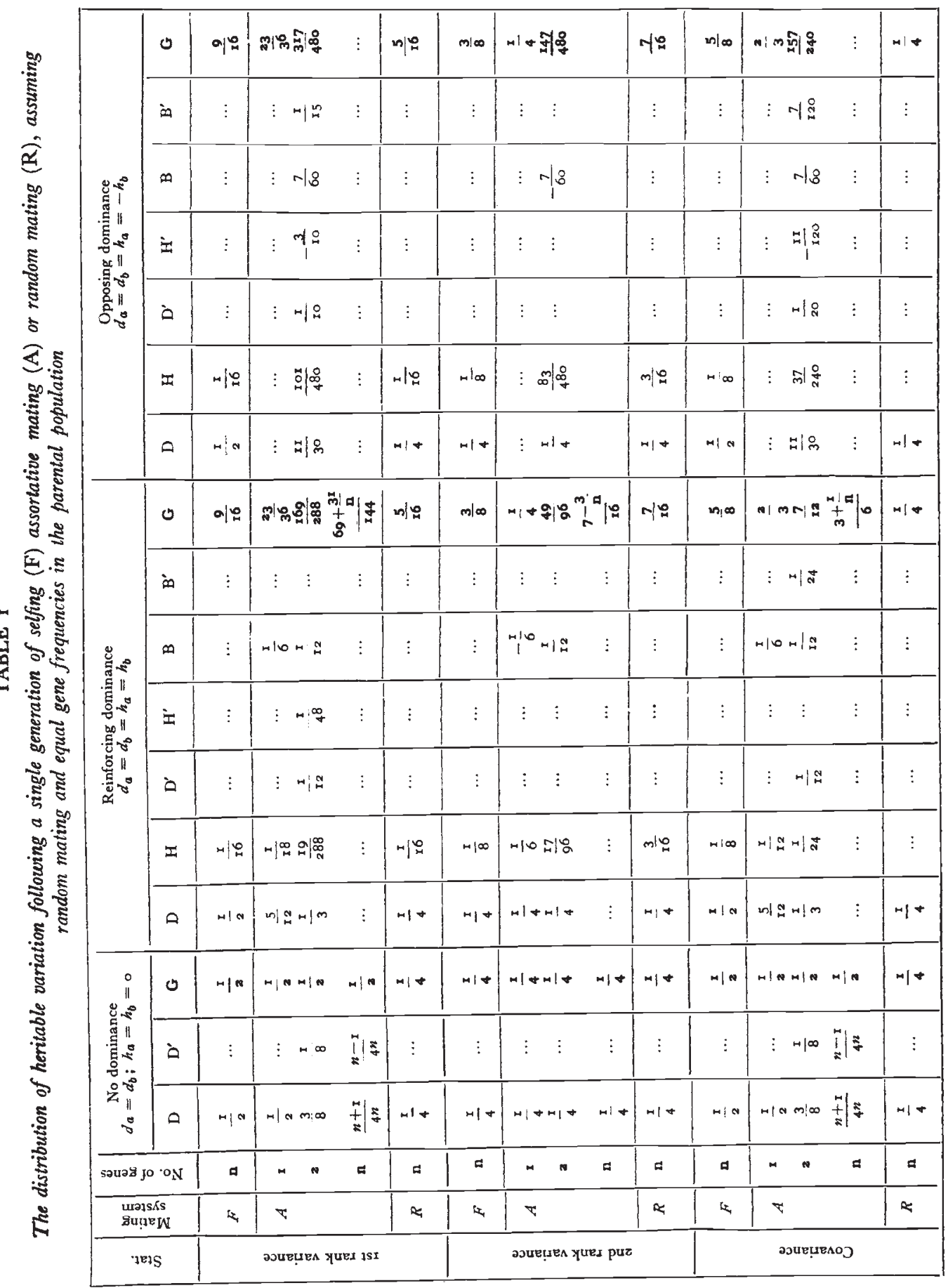


genes only are considered). This part of the variation we can denote by $\mathrm{D}^{\prime}$.

The variance of $A$ family means can now be re-expressed as

$$
\frac{n+\mathrm{I}}{4^{n}} \mathrm{D}+\frac{n-\mathrm{I}}{4^{n}} \mathrm{D}^{\prime}=\frac{1}{2} \mathrm{G} \text {. }
$$

In the same way terms may be obtained for the 2nd rank variance and the covariance.

The constitution in terms of $\mathrm{D}$ and $\mathrm{D}^{\prime}$ of the three statistics for all three mating systems are given in table $\mathrm{I}$ for $\mathrm{I}, 2$ and $n$ genes assuming no dominance. No $\mathrm{D}^{\prime}$ items occur in the $F$ and $R$ statistics, of course, since the product terms contributed by reinforcing hom/hom combinations cancel out against corresponding negative terms derived from equal proportions of opposing hom/hom combinations (i.e. $A A b b$, $a a B B$, etc.). It will be noted that although the proportion of the genetic variability exhibited by $A$ family means remains for any number of genes statistically equivalent to that obtained under selfing, its constitution in terms of $\mathrm{D}$ and $\mathrm{D}^{\prime}$ depends on the number of genes $(n)$ involved. Where $n$ is large the proportion of $\mathrm{G}$ exhibited as D is not appreciably greater for assortative than for random mating; the increased genetic variation therefore depending not on increased homozygosity but on an excess of reinforcing, and a corresponding deficiency of opposing, hom/hom combinations. Continued perfect assortative mating should thus result in an accumulation of reinforcing hom/hom combinations and finally achieve fixation of genetic variability in the free state (see also Wright, I92 I).

For all mating systems and for any number of genes the within family variances remain constant as $\frac{1}{4} \mathrm{D}$. The covariance formulæ are, however, equivalent to those of their corresponding ist rank variances and as such are valuable additional statistics for the estimation of variability components.

Since the effects of assortative mating ultimately depend on the precision whereby potential parents may be assigned to particular genotypic intramating groups, any factor influencing the expression of the character, genetic or environmental, must affect the consequences of the system. Thus, for instance, full dominance of the genes concerned, by preventing the separate classification of the heterozygote, alters the composition of intramating groups. Allowing explicitly for these effects, however, involves further assumptions regarding the properties of individual gene pairs. Expectations so derived, therefore, are likely to have but limited use in general analysis since each formulation must be applicable only to the situation as defined. Nevertheless the general implications may be discovered by taking two simple examples.

First we can arbitrarily assume that dominance is complete for all genes and that the direction is the same in each case (i.e. $d_{a}=d_{b}=$ $h_{a}=h_{b}$, etc.). Then if two genes are considered the nine possible 
genotypes of a randomly breeding population form three intramating groups when genotypic classification is complete. The expectations for all three statistics may be derived as before and are given together with the comparable $F$ and $R$ statistics in table I. The proportion of $\mathrm{G}$, given these conditions, has been calculated for $n$ genes (where $n>1$ ) by Dr Hayman of this Unit, and is also given in table 1 . Consideration of the two gene situation indicates that the increase in genetic variation of family means compared with that obtained under random mating now depends not only on $\mathrm{D}^{\prime}$ but also on $\mathrm{H}^{\prime}\left(h_{a} h_{b}\right.$, etc.) and $\mathrm{B}\left(d_{a} h_{a} ; d_{b} h_{b}\right.$, etc. $)$ items. These latter items measure the average contribution of individual genes to that proportion of the variability deriving from an excess of reinforcing heterozygotic/ heterozygotic and homozygotic/heterozygotic combinations. In addition, where the number of genes involved is large, the total genetic variation is now lower than that expected under selfing. Even so, the frequencies of genic combinations giving extreme effects are being increased at the expense of the more balanced combinations, and continued perfect assortative mating must again ultimately result in the fixation of extreme expressions of the character in question. However, compared with the situation involving no dominance, the process must be considerably retarded, at least at that end of the distribution towards which dominance is directed.

The covariance statistic, although including $\mathrm{B}^{\prime}\left(d_{b} h_{a}, d_{a} h_{b}\right.$, etc. $)$ in place of an $\mathrm{H}^{\prime}$ item, still depends for its increased value relative to the $R$ generation on items arising from reinforcing genic combinations and as such is again a useful additional statistic in estimation. The 2nd rank variance is now no longer equivalent in its constitution to that of the $R$ generation : but where $n$ is large the two statistics should not differ in value and so this statistic can play little part in comparisons between mating systems.

Finally, in table 1 , equations for the statistics have been derived for two genes where dominance is complete but opposing. The presence of positive $\mathrm{D}^{\prime}, \mathrm{B}$ and $\mathrm{B}^{\prime}$ and negative $\mathrm{H}^{\prime}$ items in the Ist rank statistics again indicates an excess of reinforcing genic combinations and hence essentially a situation similar to that observed for reinforcing dominance. By contrast, however, the and rank variance expectation, where $n=2$, is lower than is obtained under random mating; but although expectations involving larger numbers of genes have not been derived, it must be expected that as $n$ increases the nearer will the proportion of genetic variability displayed by this statistic approach that under random mating.

More complex genetical situations involving genic interaction and linkage have not been considered. But whatever the genetical control of the character, it cannot but be inferred that the continued reassociation of genes into combinations having extreme effects at the expense of more balanced combinations will remain the most important attribute of assortative mating; and while dominance, genic inter- 
action or linkage in opposition can retard the process, continued perfect assortative mating must eventually achieve fixation of the extreme expressions of the character involved unless true overdominance exists.

Although in theory the generation to generation increase in variance of $A$ families relative to $R$ families can be related to different additional components of variation, depending on the genetic control of the character, the recognition of these components separately is likely to prove difficult. With data from but a single generation available, the overall effects of assortative mating can best be measured by estimating any increase in genetic variation of $A$ families relative to that expected under random mating, using both $F$ and $R$ generations as baselines for assessment. $F$ and $R$ generations can also be employed in determining the degree of dominance and other genetic effects in the data, and any detected increase in genetic variation in the $A$ generation may then be interpreted accordingly. Doubtless some direct separation of the components entering into the increased variance could be effected by suitable experiments but so far this has not been attempted.

\section{THE EXPERIMENTS}

(i) Structure

The investigations involved the setting up of two experiments, basically of the same design but differing in the numbers of parents involved and the material used. In both instances plants used in the formation of a parental pool were individuals of $F_{2}$ populations derived from crosses between inbred lines of Nicotiana rustica. It may safely be assumed therefore that the basic individuals were fairly representative in terms of average heterozygosity of a randomly breeding population: a population, moreover, wherein each gene was represented by two allelomorphs only, each having a frequency of 0.5 .

The choice of a quantitative character, upon which the effects of assortative mating could be studied, presented little difficulty. Flowering time was the most obvious in that data needed for the construction of the mating programme could be collected early enough in the season to ensure that all crosses could be successfully completed during the same season.

In I95I, $F_{2}$ plants derived from a cross between our varieties I and 5 of Nicotiana rustica (see Mather and Vines, I953) were grown in two blocks each consisting of $5^{\circ}$ individuals. Plants were then assortatively and randomly mated on a between block basis in the following manner. Firstly the earliest flowering plant in Block I was mated to the earliest flowerer in Block II, the second earliest in Block I to the second earliest in Block II, and so on, giving a total of $5^{\circ}$ independent assortative pair matings and yielding a possible 50 families of the third generation $\left(A_{3}\right)$. Secondly, plants were mated 
according to their field position, the first plant in Block $I$ to the first in Block II, the second to the second, etc. Since individuals within the blocks were planted out at random (i.e. taken as they came from seed pans and seed boxes) field position should have been independent of genotype, and this therefore yielded 50 families derived by random mating in the third generation $\left(R_{3}\right)$. (In this instance the $R_{3}$ generation corresponds to an $\mathrm{S}_{3}$ generation under the notation of Mather and Vines.) In addition Ioo $F_{3}$ families were obtained by selfing each individual of the parental pool.

In practice, 48 families of each biparental generation and $99 F_{3}$ families were available for sowing in 1952 and these, with the parents, $F_{1}$ and $F_{2}$ were grown in a randomised two-block layout of a design similar to that devised by Mather and Vines.

The I953 experiment was initiated in $195^{2}$ using individuals of an $F_{2}$ population stemming from a cross between varieties 12 and 2 of Nicotiana rustica, but 40 plants only were used in the construction of a parental pool. Twenty $A_{3}, 20 R_{3}$ and $40 F_{3}$ families obtained by the methods described above were available for sowing in 1953 and these were grown in essentially the same experimental layout as that of 1952 .

In both cases the plants were scored for flowering time (expressed in days after 2oth June) and also for final height (in inches).

\section{(ii) Correlation between parents for flowering time and height}

An indication of the success of the methods employed in randomising and assorting the parents can be obtained by calculating the correlation coefficient $(r)$ between plants forming the male and female parental series of the $A$ and $R$ systems respectively. The appropriate $r$ values for flowering time are given in the first part of table 2 together with the probability of the departure of these values from zero. For both experiments only the $A$ male and female series show significantly high values, the $R$ values indicating nothing but true random association. In both cases, therefore, the requisite degrees of phenotypic correlation have been achieved, although in the $195^{2}$ experiment the degree of correlation $(r=0.86)$ was somewhat lower than expected.

Mather and Vines note that flowering time and height appear to be partially correlated in expression in so far as the taller plants tend to be later flowering. The between character $r$ values calculated for $120 F_{2}$ plants associated with the $195^{2}$ experiments and $100 F_{2}$ plants associated with the I953 experiments show such to be indeed the case (see table 2). Accordingly parental correlations in respect of height were found for the different mating systems in both experiments and these are also shown in table 2. It will be observed that in the $195^{2}$ experiment assorting on flowering time resulted in a significant correlation between mates with regard to height, although the strength of this correlation is considerably lower than for the primary character itself. Despite a similar correlation between height 
and flowering time as in the $195^{2}$ experimental material, parental correlation for height was not achieved in the 1953 experiment. The bias extended to height as a partially correlated character by assorting on flowering time depends, of course, in a statistical manner on the strength of correlation between characters and this correlation was sufficient to achieve a significant association of plants in respect of height in but one instance.

Analysis of the height data in addition to the flowering time data should, therefore, provide us not only with information as to the effects of different levels of assortative mating, strong and weak, but also give some indication of the degree and nature of any correlated effect which may be induced in height as a secondary correlated character.

TABLE 2

Correlation between parents for flowering time and height, 1952 and 1953

\begin{tabular}{|c|c|c|c|c|c|c|}
\hline \multirow{2}{*}{$\begin{array}{c}\text { Parental } \\
\text { correlation } \\
\text { between }\end{array}$} & \multicolumn{3}{|c|}{$195^{2}$} & \multicolumn{3}{|c|}{1953} \\
\hline & $\begin{array}{c}\text { Correlation } \\
\text { coeff. }(r)\end{array}$ & $\mathbf{N}$ & $\begin{array}{c}\text { Dev. from o } \\
\qquad P\end{array}$ & $\begin{array}{c}\text { Correlation } \\
\text { coeff. }(r)\end{array}$ & $\mathbf{N}$ & Dev. from o \\
\hline \multicolumn{7}{|c|}{ Flowering time } \\
\hline $\begin{array}{l}A \delta \text { and } q \text { series } \\
R \delta \text { and } \phi \text { series }\end{array}$ & $\begin{array}{r}0.86 \\
-0.09\end{array}$ & $\begin{array}{l}46 \\
46\end{array}$ & $\begin{array}{c}<0 \cdot 001 \\
\ldots\end{array}$ & $\begin{array}{l}0.97 \\
0.29\end{array}$ & $\begin{array}{l}18 \\
18\end{array}$ & $\begin{array}{l}<0 \cdot 001 \\
>0 \cdot 1\end{array}$ \\
\hline \multicolumn{7}{|c|}{ Height } \\
\hline $\begin{array}{l}A \delta^{*} \text { and } \phi \text { series } \\
R \delta^{*} \text { and } \phi \text { series }\end{array}$ & $\begin{array}{l}0.38 \\
0 \cdot 18\end{array}$ & $\begin{array}{l}46 \\
46\end{array}$ & $\begin{array}{l}0.01-0.001 \\
>0 \cdot 1\end{array}$ & $\begin{array}{l}0 \cdot 19 \\
0.00\end{array}$ & $\begin{array}{l}18 \\
18\end{array}$ & $\begin{aligned}>0 \cdot 1 \\
\\
\ldots\end{aligned}$ \\
\hline \multicolumn{7}{|c|}{ Height and flowering time } \\
\hline $\begin{array}{c}\text { Height and } \\
\text { flowering time }\end{array}$ & 0.45 & II 8 & $<0 \cdot 001$ & $0 \cdot 47$ & 98 & $<0 \cdot 001$ \\
\hline
\end{tabular}

\section{ANALYSIS OF VARIABILITY}

(i) General

For each of the two characters and each of the three mating systems, three second degree statistics have been calculated and are given in tables 4 and 5 together with their compositions in terms of $\mathrm{G}$ and $\mathrm{E}$ (the environmental component), assuming no dominance. The notation devised by Mather and Vines (1953) to denote the different statistics has been extended to cover present requirements and is presented in table 3 .

The direct estimates of $E_{1}$ and $E_{2}$ were obtained by the methods of Mather and Vines except in the case of the $E_{2} \mathrm{~S}$ of 1953 . In this 
year $E_{2} \mathrm{~s}$ were obtained from $F_{2}$ plot means and not $F_{1}$ and parental plot means. It was considered that this would allow not only for

TABLE 3

Second degree statistics calculated for progenies

\begin{tabular}{|c|c|}
\hline Notation & \\
\hline $\begin{array}{l}\mathrm{V}_{1 F_{3}} \\
\mathrm{~V}_{2 F_{3}} \\
\mathrm{~W}_{1 F_{23}}\end{array}$ & $\begin{array}{l}\text { Variance of means of families derived by selfing } \\
\text { Mean variance of families derived by selfing } \\
\text { Covariance of family means and parental values }\end{array}$ \\
\hline $\begin{array}{l}\mathrm{V}_{1 A_{3}} \\
\mathrm{~V}_{2 A_{3}} \\
\mathrm{~W}_{1 . A_{23}}\end{array}$ & $\begin{array}{l}\text { Variance of means of assortative biparental progenies } \\
\text { Mean variance of assortative biparental progenies } \\
\text { Covariance of the family means and the means of the parental pair } \\
\text { values }\end{array}$ \\
\hline $\left.\begin{array}{l}V_{1 R_{3}} \\
V_{2 R_{3}} \\
W_{1 R_{23}}\end{array}\right\}$ & $\begin{array}{l}\text { Analogous to above statistics but for the progenies of random bi- } \\
\text { parental matings }\end{array}$ \\
\hline
\end{tabular}

TABLE 4

Results-height and flowering time-1952

\begin{tabular}{|c|c|c|c|c|c|}
\hline \multirow{2}{*}{ Statistic } & \multirow{2}{*}{$\begin{array}{c}\text { Composition } \\
(\mathrm{H}=\mathrm{o})\end{array}$} & \multicolumn{3}{|c|}{ Observed } & \multirow{2}{*}{ Expected } \\
\hline & & I & II & Mean & \\
\hline \multicolumn{6}{|c|}{ Flowering time } \\
\hline $\begin{array}{l}\mathrm{E}_{1} \\
\mathrm{E}_{2} \\
\mathrm{~V}_{1 F_{3}} \\
\mathrm{~V}_{2 F_{3}} \\
\mathrm{~W}_{1 F_{23}} \\
\mathrm{~V}_{1 A_{2}} \\
\mathrm{~V}_{2} A_{2} \\
\mathrm{~W}_{1 A_{23}} \\
\mathrm{~V}_{1 R_{3}} \\
\mathrm{~V}_{2 R_{3}} \\
\mathrm{~W}_{1} R_{23}\end{array}$ & $\begin{array}{c}E_{1} \\
E_{2} \\
\frac{1}{2} G+E_{2} \\
\frac{1}{4} G+E_{1} \\
\frac{1}{2} G \\
\frac{1}{2} G+E_{2} \\
\frac{1}{4} G+E_{1} \\
\frac{1}{2} G \\
\frac{1}{4} G+E_{2} \\
\frac{1}{4} G+E_{1} \\
4 G\end{array}$ & $\begin{array}{r}5 \cdot 2449 \\
2 \cdot 1004 \\
34 \cdot 2642 \\
25 \cdot 2120 \\
12 \cdot 0108 \\
21 \cdot 7435 \\
42 \cdot 5375 \\
7 \cdot 9965 \\
14 \cdot 8714 \\
27 \cdot 9854 \\
6 \cdot 3074\end{array}$ & $\begin{array}{r}7 \cdot 3301 \\
4 \cdot 5154 \\
25 \cdot 2843 \\
24 \cdot 0040 \\
12 \cdot 5519 \\
23 \cdot 0494 \\
43 \cdot 4267 \\
10.6846 \\
16.4438 \\
28 \cdot 0000 \\
5 \cdot 1554\end{array}$ & $\begin{array}{r}6 \cdot 2875 \\
3 \cdot 3079 \\
29 \cdot 7743 \\
24 \cdot 6080 \\
12 \cdot 2814 \\
22 \cdot 3965 \\
42 \cdot 9821 \\
9 \cdot 3406 \\
15 \cdot 6576 \\
27 \cdot 9927 \\
5 \cdot 7314\end{array}$ & $\begin{array}{r}18 \cdot 3784 \pm 0 \cdot 8767 \\
7 \cdot 6888 \pm 0 \cdot 9516 \\
26.5941 \pm 1 \cdot 0364 \\
27.8310 \pm 0 \cdot 7882 \\
18 \cdot 9053 \pm 1 \cdot 0860 \\
19.7130 \pm 1 \cdot 1967 \\
27.8310 \pm 1 \cdot 0158 \\
12.0242 \pm 1 \cdot 1967 \\
17 \cdot 1414 \pm 0 \cdot 8336 \\
27.8310 \pm 0 \cdot 7882 \\
9.4526 \pm 0.5430\end{array}$ \\
\hline \multicolumn{6}{|c|}{ Height } \\
\hline $\begin{array}{l}\mathrm{E}_{1} \cdot \\
\mathrm{E}_{2} \\
\mathrm{~V}_{1 F_{3}} \\
\mathrm{~V}_{2 F_{8}} \\
\mathrm{~W}_{1 F_{23}} \\
\mathrm{~V}_{1 A_{3}} \\
\mathrm{~V}_{2 A_{3}} \\
\mathrm{~W}_{1 A_{23}} \\
\mathrm{~V}_{1 R_{3}} \\
\mathrm{~V}_{2 R_{3}} \\
\mathrm{~W}_{1 R_{33}}\end{array}$ & $\begin{array}{l}\cdot \\
: \\
: \\
: \\
: \\
: \\
: \\
:\end{array}$ & $\begin{array}{r}4 \cdot 8937 \\
3 \cdot 8950 \\
23 \cdot 3099 \\
20 \cdot 4859 \\
12 \cdot 3073 \\
16.9347 \\
16.5208 \\
8 \cdot 4733 \\
10 \cdot 5538 \\
17 \cdot 1417 \\
7 \cdot 4326\end{array}$ & $\begin{array}{r}5 \cdot 7968 \\
4 \cdot 6749 \\
22 \cdot 3106 \\
17 \cdot 6428 \\
12 \cdot 6316 \\
18 \cdot 4715 \\
26 \cdot 3611 \\
7 \cdot 8085 \\
14.8513 \\
21 \cdot 8146 \\
7 \cdot 6251\end{array}$ & $\begin{array}{r}5 \cdot 3453 \\
4 \cdot 2850 \\
22 \cdot 8103 \\
19 \cdot 0644 \\
12 \cdot 4695 \\
17 \cdot 7031 \\
21 \cdot 4410 \\
8 \cdot 1409 \\
12 \cdot 4026 \\
19 \cdot 4782 \\
7 \cdot 5289\end{array}$ & $\begin{array}{r}10 \cdot 5030 \pm 1 \cdot 0045 \\
6 \cdot 0749 \pm 1 \cdot 1337 \\
21 \cdot 6200 \pm 1 \cdot 2347 \\
18 \cdot 2755 \pm 0 \cdot 9390 \\
15.5451 \pm 1 \cdot 2938 \\
15.9594 \pm 1 \cdot 4257 \\
18 \cdot 2755 \pm 1 \cdot 2102 \\
9 \cdot 945 \pm 1 \cdot 4257 \\
13.8474 \pm 0.9932 \\
18.2755 \pm 0.9390 \\
7.7726 \pm 0.6469\end{array}$ \\
\hline
\end{tabular}

field variation but also to some extent for sampling variation entailed in the use of segregating families. Doubtless a more satisfactory 
method would have been to have used $\mathrm{E}_{2} \mathrm{~s}$ estimated in the normal manner and to have introduced a separate term for sampling variation based on the numbers of plants per plot and the within family variance: but at the time it was not considered that the extra computation involved would have been justified.

Information as to the change in heritable variation associated with different mating systems could be obtained by a modified form of offspring/parent regression analysis. But more useful and more

TABLE 5

Results-height and flowering time-1953

\begin{tabular}{|c|c|c|c|c|}
\hline \multirow{2}{*}{ Statistic } & \multicolumn{3}{|c|}{ Observed } & \multirow{2}{*}{ Expected } \\
\hline & I & II & Mean & \\
\hline \multicolumn{5}{|c|}{ Flowering time } \\
\hline $\begin{array}{l}\mathrm{E}_{1} \\
\mathrm{E}_{2} \\
\mathrm{~V}_{1 F_{3}} \\
\mathrm{~V}_{2 F_{3}} \\
\mathrm{~W}_{1 F_{23}} \\
\mathrm{~V}_{1 A_{2}} \\
\mathrm{~V}_{2 A_{3}} \\
\mathrm{~W}_{1 A_{23}} \\
\mathrm{~V}_{1 R_{3}} \\
\mathrm{~V}_{2 R_{2}} \\
\mathrm{~W}_{1 R_{33}}\end{array}$ & $\begin{array}{r}24 \cdot 9895 \\
25 \cdot 5500 \\
127 \cdot 0143 \\
74 \cdot 6950 \\
80 \cdot 8909 \\
85 \cdot 6994 \\
68 \cdot 7700 \\
63 \cdot 7726 \\
94 \cdot 8475 \\
77 \cdot 0900 \\
56 \cdot 8245\end{array}$ & $\begin{array}{r}36 \cdot 8400 \\
45 \cdot 1980 \\
136 \cdot 9804 \\
65 \cdot 9225 \\
78 \cdot 9900 \\
76 \cdot 3506 \\
94 \cdot 4100 \\
68 \cdot 3308 \\
71 \cdot 5815 \\
111 \cdot 6950 \\
50 \cdot 0832\end{array}$ & $\begin{array}{r}30 \cdot 9148 \\
35 \cdot 3740 \\
131 \cdot 9974 \\
70 \cdot 3088 \\
79 \cdot 9405 \\
81 \cdot 0250 \\
81 \cdot 5900 \\
66 \cdot 0517 \\
83 \cdot 2145 \\
94 \cdot 3925 \\
53 \cdot 4539\end{array}$ & $\begin{array}{r}34 \cdot 6627 \pm 4 \cdot 8571 \\
34 \cdot 1481 \pm 5 \cdot 271 \\
126 \cdot 5178 \pm 9 \cdot 7473 \\
80 \cdot 8475 \pm 6 \cdot 7980 \\
92 \cdot 3697 \pm 6 \cdot 0162 \\
90 \cdot 6124 \pm 9 \cdot 9767 \\
80 \cdot 8475 \pm 6 \cdot 7980 \\
56 \cdot 4643 \pm 6 \cdot 6295 \\
80 \cdot 3329 \pm 7 \cdot 2355 \\
80 \cdot 8475 \pm 6 \cdot 7980 \\
46 \cdot 1848 \pm 3 \cdot 0081\end{array}$ \\
\hline \multicolumn{5}{|c|}{ Height } \\
\hline $\begin{array}{l}\mathrm{E}_{1} \\
\mathrm{E}_{2} \\
\mathrm{~V}_{1 F_{3}} \\
\mathrm{~V}_{2 F_{3}} \\
\mathrm{~W}_{1 F_{32}} \\
\mathrm{~V}_{1 A_{2}} \\
\mathrm{~V}_{2 A_{2}} \\
W_{1 A_{32}} \\
\mathrm{~V}_{1 R_{2}} \\
\mathrm{~V}_{2 R_{2}} \\
W_{1 R_{23}}\end{array}$ & $\begin{array}{l}13 \cdot 4000 \\
19 \cdot 0898 \\
68 \cdot 6203 \\
52 \cdot 5125 \\
28 \cdot 7753 \\
61 \cdot 6988 \\
63 \cdot 5650 \\
11 \cdot 2424 \\
60 \cdot 4842 \\
80 \cdot 4250 \\
11 \cdot 3737\end{array}$ & $\begin{array}{r}19 \cdot 0347 \\
21 \cdot 7900 \\
92 \cdot 7091 \\
64 \cdot 5425 \\
30 \cdot 4814 \\
45 \cdot 5600 \\
92 \cdot 4350 \\
16 \cdot 1868 \\
30 \cdot 7604 \\
101 \cdot 2450 \\
9 \cdot 4379\end{array}$ & $\begin{array}{l}16 \cdot 2174 \\
20 \cdot 4399 \\
80 \cdot 6647 \\
58 \cdot 5275 \\
29 \cdot 6284 \\
53 \cdot 6294 \\
78 \cdot 0000 \\
13 \cdot 7146 \\
45 \cdot 6223 \\
90 \cdot 8350 \\
10 \cdot 4058\end{array}$ & $\begin{array}{l}44 \cdot 5332 \pm 4 \cdot 8195 \\
28 \cdot 925^{1} \pm 5 \cdot 231 \\
72 \cdot 55^{6} \pm 9 \cdot 6720 \\
66 \cdot 3487 \pm 6 \cdot 7454 \\
43 \cdot 6310 \pm 5 \cdot 9697 \\
48 \cdot 1345 \pm 9 \cdot 9996 \\
66 \cdot 3487 \pm 6 \cdot 7454 \\
19 \cdot 2094 \pm 6 \cdot 578 \\
50 \cdot 7406 \pm 7 \cdot 1796 \\
66 \cdot 3487 \pm 6 \cdot 7454 \\
21 \cdot 8155 \pm 2 \cdot 9848\end{array}$ \\
\hline
\end{tabular}

accurate information can be obtained by methods allowing the simultaneous utilisation of all the possible second degree statistics and this has been accomplished by the methods first discussed by Mather ( $1949 a$ and $b$ ). An added advantage is that the methods depend mainly on the use of 3 rd generation statistics based on large numbers of progeny and place no weight on statistics derived solely from small numbers of parents. At the same time they allow the detection of dominance and interaction as disturbing influences on the estimation and interpretation of the components of variation. 
As previously noted we can best test the hypotheses outlined by comparing the total genetic variation obtained under each mating system. The first analyses assume the absence of dominance and reference to table I indicates that under these conditions only the Ist rank statistics of the $A$ generation are inflated by $D^{\prime}$ items. Since progenies derive from a common parental population the total potential genetic variation (G) must be a constant for all mating systems. Consequently the proportion of $\mathrm{G}$ appearing in the $A$ Ist rank statistics can be estimated relative to the proportions appearing in all the remaining statistics. This would be most directly accomplished by allowing the coefficient of $\mathrm{G}$ for the $A$ ist rank statistics $\left(a_{A}\right)$ to take its own value while allotting to the remaining statistics the coefficients fixed by hypothesis. But in point of fact the estimation

TABLE 6

Values of components and estimated coefficients of $V_{1 A_{3}}$ and $W_{1 R_{23}}$ (i.e. $a_{A}$ )

\begin{tabular}{|c|c|c|c|c|}
\hline & \multicolumn{2}{|c|}{ Flowering time } & \multicolumn{2}{|l|}{ Height } \\
\hline & I 952 & 1953 & 1952 & I 553 \\
\hline $\begin{array}{l}G \\
G_{1} \\
E_{1} \\
E_{2}\end{array}$ & $\begin{array}{c}37 \cdot 81 \pm 2 \cdot 17(31 \cdot 30 \pm 2 \cdot 46) \\
24 \cdot 05 \pm 2 \cdot 39(22 \cdot 65 \pm 2 \cdot 59) \\
18 \cdot 38 \pm 0 \cdot 88(18 \cdot 48 \pm 1 \cdot 33) \\
7 \cdot 69 \pm 0 \cdot 95(9 \cdot 08 \pm 1 \cdot 04)\end{array}$ & $\begin{array}{r}184 \cdot 74 \pm 12 \cdot 03 \\
112 \cdot 93 \pm 13 \cdot 26 \\
34 \cdot 66 \pm 4 \cdot 86 \\
34 \cdot 15 \pm 5 \cdot 27\end{array}$ & $\begin{array}{r}31 \cdot 09 \pm 2 \cdot 59(28 \cdot 3 \mathrm{I} \pm 1 \cdot 78) \\
19.77 \pm 2 \cdot 85(19 \cdot 17 \pm 1.87) \\
10.50 \pm 1 \cdot 04(12.19 \pm 0.97) \\
6 \cdot 07 \pm 1 \cdot 13(6.67 \pm 0.75)\end{array}$ & $\begin{array}{l}87 \cdot 26 \text { 士1 } 1 \cdot 94 \\
38 \cdot 24 \pm 13 \cdot 16 \\
44 \cdot 53 \pm 4 \cdot 82 \\
28 \cdot 93 \pm 5 \cdot 23\end{array}$ \\
\hline $\begin{array}{l}\mathrm{G}_{1} / \mathrm{G} \\
a_{A}\end{array}$ & $\begin{array}{ll}0.64 \pm 0.07 & \left(0.7^{2} \pm 0.09\right) \\
0.32 \pm 0.035 & (0.36 \pm 0.045)\end{array}$ & $\begin{array}{l}0.61 \pm 0.08 \\
( \pm 0.01) \\
0.31 \pm 0.038 \\
( \pm 0.005)\end{array}$ & $\begin{array}{l}0.64 \pm 0.10 \quad(0.68 \pm 0.07) \\
0.3^{2} \pm 0.048 \quad(0.34 \pm 0.035)\end{array}$ & $\begin{array}{l}0.44 \pm 0 \cdot 10 \\
( \pm 0 \cdot 10) \\
0.22 \pm 0.050 \\
( \pm 0.050)\end{array}$ \\
\hline & & $a_{F}=0$ & $=0.25$ & \\
\hline
\end{tabular}

was achieved by retaining the theoretical $\alpha_{A}$ value of $\frac{1}{2}$ and allowing $\mathrm{G}$ itself to assume its own value. The formula for $\mathrm{V}_{1 A 3}$ and $\mathrm{W}_{1 A 23}$ now become $\frac{1}{2} G_{1}+E_{2}$ and $\frac{1}{2} G_{1}$ respectively, $G_{1}$ thus being a distinct variable in the least squares equations. $\alpha_{A}$ is then obtained as $\frac{1}{2}\left(\frac{G_{1}}{G}\right)$ where $G$ is estimated from the remaining statistics jointly. Since in this type of analysis factors affecting the value of $\mathrm{G}$ are indistinguishable from those affecting the coefficients of $G$ (see also Bateman and Mather, $195^{\mathrm{I}}$ ), this final estimate of $\alpha_{A}$ is the same whether $\mathrm{G}$ or its coefficient is allowed to vary.

The least squares estimates of the four components $G, G_{1}, E_{1}$ and $\mathrm{E}_{2}$, calculated from the means of blocks, for both characters and both experiments are given in table 6 . Values of the components can also be found for blocks separately and the values may be substituted 
into the formulæ to give expectations for the statistics for each block and the block means. The deviations of observed from expected can then be analysed in the way described by Mather and Vines. The final results of the analyses are given in table 7 .

The strict error variation which is obtained from differences between duplicate estimates of the same statistic (one from each block) has been partitioned into the two heterogeneity items shown; but since in no instance are the two items sufficiently different to merit separate consideration the appropriate duplicate M.S. is taken as the basic error in all cases. The residual disturbances item measures

TABLE 7

Analysis of variation for height and flowering time

\begin{tabular}{|c|c|c|c|c|c|c|c|}
\hline \multirow{2}{*}{ Item } & \multicolumn{4}{|c|}{$195^{2}$} & \multicolumn{3}{|c|}{ ז953 } \\
\hline & $\mathrm{N}$ & SS & MS & $\mathbf{P}$ & SS & MS & $\mathrm{P}$ \\
\hline \multicolumn{8}{|c|}{ Flowering time } \\
\hline $\begin{array}{l}\text { Residual interaction } \\
\text { Heterogeneity of } \\
\text { duplicates- } \\
\text { Residual interaction } \\
\text { Components }\end{array}$ & $\begin{array}{l}7 \\
4 \\
\end{array}$ & $\begin{array}{r}979 \cdot 59 \\
\\
33 \cdot 35 \\
19 \cdot 69\end{array}$ & $\begin{array}{l}4 \cdot 76 \\
4 \cdot 92 \\
\end{array}$ & $\ldots$ & $\begin{array}{r}1115.72 \\
512.39\end{array}$ & $\begin{array}{l}21 \mathrm{II} \cdot 46 \\
\\
159 \cdot 39 \\
128 \cdot 10\end{array}$ & $\ldots$ \\
\hline Total & 18 & $1032 \cdot 63$ & $\ldots$ & $\ldots$ & $3^{108.35}$ & $\ldots$ & $\ldots$ \\
\hline \multicolumn{8}{|c|}{ Height } \\
\hline $\begin{array}{l}\text { Residual interaction . } \\
\text { Heterogeneity of } \\
\text { duplicates- } \\
\text { Residual interaction } \\
\text { Components }\end{array}$ & $\begin{array}{l}7 \\
4\end{array}$ & $\begin{array}{l}120 \cdot 44 \\
\\
5 \mathrm{I} \cdot 46 \\
23 \cdot 84\end{array}$ & $\begin{array}{l}7 \cdot 35 \\
5 \cdot 96\end{array}$ & $0 \cdot 2-0 \cdot I$ & $\begin{array}{l}867 \cdot 29 \\
735 \cdot 74\end{array}$ & $\begin{array}{r}123.90 \\
183.94\end{array}$ & $0.05-0.01$ \\
\hline Total & 18 & $195 \cdot 74$ & $\cdots$ & $\ldots$ & $5900 \cdot 77$ & $\ldots$ & $\ldots$ \\
\hline
\end{tabular}

the departure of the observed mean value of each statistic from that expected under the conditions of no dominance, etc., outlined, and will thus include differences ascribable to dominance, genic interaction and linkage. It will be observed that only for flowering time in 1952 and for height in 1953 is there good evidence of one or more of these agencies affecting the expression of the character. In the other two cases, therefore, we can assume that the value of $a_{A}$ derived from the ratio $\frac{G_{1}}{G}$ will be affected only by the degree of phenotypic correlation achieved in the $A$ parents and the extent to which genotypic misclassification has resulted from environmental fluctuations. 
The ratios $\frac{\mathrm{G}_{\mathbf{1}}}{\mathrm{G}}$ and the values of $\alpha_{A}$ estimated from them are given in table 6 . In all cases the standard errors attached to the components and to the ratios have been calculated using as basic error the duplicate M.S. weighted by means of the appropriate elements of the $c$-matrix used in the estimation of the components.

Consider first the results for I952. In flowering time and height the values for $\alpha_{A}$, in both cases 0.323 are higher than the theoretical $\alpha_{R}$ value of 0.25 , but only in the case of flowering time is the error sufficiently low for significance to be approached $(P=0 \cdot 1-0.05)$. The values are, in both instances, significantly lower than that expected under complete genotypic correlation. Thus, compared with the response achieved in height with a relatively weak parental correlation, the response obtained in flowering time is disappointing. Doubtless this can in large measure be related to the presence of dominance, interaction or linkage demonstrable in the flowering time analyses but in order to observe whether any individual statistic was having an undue effect in the estimation of $\frac{G_{1}}{G}$ ratios, standard errors have been calculated for the expected values of the statistics. Both the expected values and the standard errors are given in table 4 .

It becomes apparent that two statistics show extreme deviation from expected. These are $\mathrm{V}_{2 A 3}$ and the direct estimate of $\mathrm{E}_{1}$. Of course, in so far as $\mathrm{V}_{2 A 3}$ comprises a term for $\mathrm{E}_{1}$ any sharp deviation in the former must affect the estimation of the latter. But why $\mathrm{V}_{2 A 3}$ should be almost double the values observed for the other and rank variances is not immediately clear. Reference to table I shows that the genetic variance of the 2nd rank statistic is inflated for $A$ generations relative to $F$ and $R$ generations by reinforcing dominance particularly where the numbers of genes involved are small. But this could hardly account for the large differences observed unless certain forms of genic interaction yield additional inflationary components of variation for this statistic under a system of assortative mating. Until further information is available on this point it would seem desirable to confine the estimation of $G$ to the statistics available from the $F$ and $R$ generations.

Accordingly, for flowering time in 1952 , the four components have been re-estimated, omitting $\mathrm{V}_{2 A 3}$ and also the direct estimate of $\mathrm{E}_{\mathbf{1}}$. Although for the $195^{2}$ height data deviations in these two statistics are not nearly so marked, $\mathrm{V}_{2 A 3}$ is still suspiciously high, and to keep the analyses comparable the components have been recalculated on the same basis as for flowering time. The new values for components and ratios together with standard errors are given in brackets in table 6 . In both flowering time and height $\alpha_{A}$ values are now significantly higher than the theoretical $a_{R}$ value $\left(\mathrm{P}=0.05^{-0.02}\right.$ in both cases).

Both characters therefore show increased genetic variability 
relative to that obtained under random mating. In neither case is the increase a large one $\left(\alpha_{A}=0.34\right.$ as opposed to an expected 0.5$)$ but for height this must be expected since the correlation between parents was by no means great $(r=0.38)$, and as we shall see later the apparently small increase demonstrated for flowering time can be related to the greater complexity of its genetical control.

Turning to the 1953 results it can be seen that only in the case of flowering time is there any indication that $\alpha_{A}$ is greater than $\alpha_{R}$. The standard errors, shown out of brackets, calculated by the methods employed for the $195^{2}$ analyses are too high to give any significance to this difference $\left(t_{11}=\mathrm{I} \cdot 5^{8}\right.$ and $\left.\mathrm{P}=0 \cdot 2-0 \cdot \mathrm{I}\right)$. The individual block values of the components demonstrate, however, that the values of $G$ and $G_{1}$ vary but little from block to block. Standard errors, calculated using the duplicate M.S. as the basic error, are in fact inflated by large variation in $E_{1}$. Since we are concerned solely with variation in $G$ and $G_{1}$ it is permissible to calculate the ratio $G / G_{1}$ for blocks separately and then estimate the standard error of the mean as $\sqrt{\frac{1}{2} \Sigma d e v^{2}}$. For the two blocks the ratios are $\frac{115 \cdot 73}{187 \cdot 03}=0 \cdot 62$ and $\frac{110.13}{182.45}=0.60$, and the standard error of the mean 0.61 is then 0.01 . Based on this error the difference $\alpha_{A}-\alpha_{R}$ approaches significance although based on but I d.f. $\left(t=\mathrm{II}_{\mathrm{I}} \cdot \mathrm{O}_{(1)}\right.$ and $\left.\mathrm{P} \bumpeq 0 \cdot 05\right)$.

In height, as we should expect in so far as no significant phenotypic correlation could be demonstrated for the parents, the estimated $A$ coefficient of $\mathrm{G}$ is in good agreement with that fixed for a random mating series (i.e. $\alpha_{A}=\alpha_{R}$ ).

Compared with the 1952 results the $a_{A}$ value for flowering time is disappointingly low when it is remembered that in this experiment the genes controlling this character appear to be wholly additive in effect. The smaller number of plants used in the formation of a parental pool (40 as opposed to 100) must, however, involve a considerably higher sampling error as a limiting feature in the response expected.

So far we have not allowed for the effects of dominance or other genetic disturbances but the analyses suggest that, for the $195^{2}$ flowering time and the 1953 height, such agencies are affecting the expression of the character. For the 1952 flowering time data, therefore, it would be useful to try and establish the nature of these agencies and at the same time attempt some assessment of their effects on the assortative mating system.

\section{(ii) Dominance analysis of the 1952 flowering time dato}

The data obtained for the $F$ and $R$ generations may be subjected to analysis allowing explicitly for, and hence estimating, the effects of dominance. Since formulation of similar expectations for the $A$ statistics involve arbitrary assumptions as to the direction and 
magnitude of dominance, these have been omitted from the analyses. The method of analysis is precisely that of Mather and Vines and so only final results are considered. The values of the four components $D, H, E_{1}$ and $E_{2}$ for flowering time in $195^{2}$ are given in table 8, together with the final analysis : the standard errors of the components have been calculated in the usual manner, using the duplicate M.S. as the basic error since the heterogeneity items are of the same order and can thus be pooled as a single error item.

The analyses give good evidence of significant dominance $(\mathrm{P}=$ $0.0 \mathrm{I}-0.00 \mathrm{I})$ and some indication that $\mathrm{H}>\mathrm{D}(\mathrm{P}=0 \cdot \mathrm{I}-0.05)$. It will also be noted that the item for residual interaction is significantly high, indicating disturbances caused by genic interaction and/or linkage. Now Hayman (unpub.) claims to have shown that where

TABLE 8

Analysis of variation in flowering time, $195^{2}$ (F and $\mathbf{R}$ statistics)

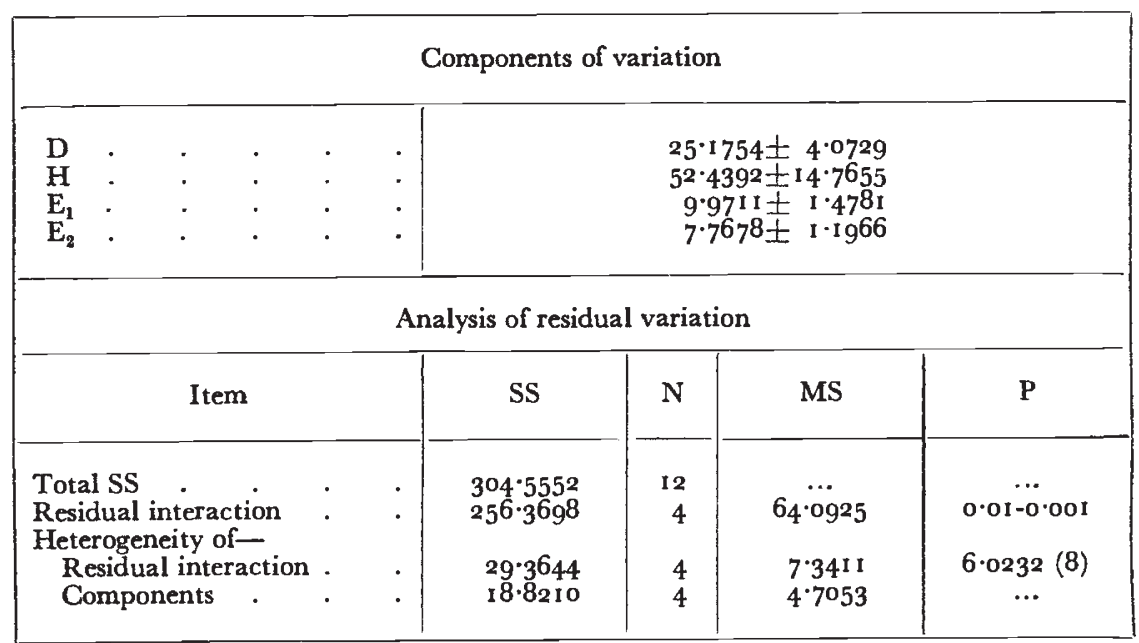

the numbers of interacting genes are small and the generation number low, genic interaction inflates $\mathrm{H}$ relative to $\mathrm{D}$. Hence the high $\mathrm{H}$ value combined with the significant residual interaction item suggests that in addition to dominance there is a certain amount of genic interaction exhibited by the data. Since Mather and Vines have found linkage between the genes controlling flowering time in this cross, the high M.S. for residual interaction may also in part be attributable to disturbances caused by linkage.

The analyses indicate, therefore, that in the $195^{2}$ experiment the genetic control of flowering time was much more complex than that of height and the estimation of $\alpha_{A}$ for flowering time assuming simple additivity is not strictly valid. Obtaining an unbiased estimate of $\alpha_{A}$ under these conditions of high dominance, and possibly genic interaction, is not, however, likely to prove easy since.some knowledge 
of the properties of individual gene pairs is first required. In the present data the $H / D$ ratio indicates at least full dominance of the majority of the genes concerned and the behaviour of the $F_{1}$ relative to the parents suggests that the dominance is preponderantly in the direction of early flowering. Neglecting any genic interaction we may argue that this most nearly approaches the first of the hypothetical situations earlier considered, where dominance was assumed to be complete and unidirectional for all genes. Reference to table I demonstrates that where $n$ is large the proportions of the total genetic variations expected for $\mathrm{V}_{1 A 3}$ and $\mathrm{W}_{1 A 23}$ under these conditions approximate to $\frac{23}{48} \mathrm{G}$ and $\frac{1}{2} \mathrm{G}$ respectively, while $\mathrm{G}$ expectations for the $F$ and $R$ statistics can be correspondingly modified to allow for $\mathrm{H}$. Then if $\mathrm{V}_{2 A 3}$ and $\mathrm{E}_{1}$ are again omitted and the $\frac{\mathrm{I}}{48}$ difference between the ist rank $A$ statistics ignored, a new inverse matrix derived as for the previous 1952 analysis may be employed to give fresh values for the four components and so enable the recalculation of the ratio $G_{1} / G$.

The value of $a_{A}$ calculated from this ratio is $0.43 \pm 0.017$. This is significantly higher than the theoretical coefficient of the $V_{1 R 3}$ statistic of $0.3 \mathrm{I} \quad\left(t_{9}=7.06\right.$ and $\left.\mathrm{P}<0.00 \mathrm{I}\right)$, clearly indicating an increased genetic variance for the $A$ families. It is, however, significantly lower than the theoretical $\alpha_{A}$ value taken as $0.4^{8}$ (i.e. the $\mathrm{V}_{1 A 3}$ coefficient) $\left(t_{(9)}=2.9 \mathrm{I}\right.$ and $\left.\mathrm{P}=0.02-\mathrm{O} \cdot \mathrm{OI}\right)$, but it approximates nearer to theoretical expectation than when dominance is ignored; and when it is remembered that the phenotypic correlation between the parents was incomplete $(r=0.85)$ this could be adjudged a satisfactory fit with expectation. At the same time it is likely that genic interaction, different dominance relationships of the genes and possibly linkage are additional variables affecting the estimation of this value for $\alpha_{A}$.

This brief treatment of the data allowing specifically for dominance serves mainly to emphasise the difficulties involved in considering its effects on a system of assortative mating. Nevertheless the method employed could well provide a basis for further research into the matter and could also be modified to allow for the effects of genic interaction and linkage. But before this can proceed with any prospect of success there is need for more accurate methods of estimating the genetic components of variation.

\section{DISCUSSION}

Consider first the results for flowering time, the assorted character. In both experiments the analyses have enabled us to demonstrate that the proportion of the potential genetic variability detectable in an $A$ generation (measured by $\alpha_{A}$ ) is significantly greater than that 
obtained under random mating $\left(\alpha_{R}\right)$. As we have seen (see Section 2), assuming the number of genes controlling the character to be large, this increased genetic variation cannot be ascribed to increased homozygosity as in the case of a selfed generation. The difference $a_{A}-a_{R}$, which for convenience we may denote by $\mathrm{G}$, is founded rather upon non-random association of the genes in such a way that an excess of reinforcing genic combinations is obtained at the expense of the more balanced (opposing) combinations. Where the genes are additive in effect (and with no dominance) the difference may be related entirely to an excess of reinforcing hom/hom combinations, and $\mathrm{C}$ will be a measure of the $\mathrm{D}^{\prime}$ item of table $\mathrm{I}$. Where, however, the genetic control of the character is more complex, the increased variance will depend also on reinforcing hom/het and het/het combinations. Subject to the dominance relationships of the genes, $\mathrm{C}$ will then measure the combined effects of the $\mathrm{D}^{\prime}, \mathrm{H}^{\prime}, \mathrm{B}$ and $\mathrm{B}^{\prime}$ items of table I. It will also measure other non-specified items if nonallelic interaction is operative. Measuring the contribution by each of the constituent items of $\mathrm{C}$ is, however, not likely to be an easy matter. In any case it would require a much more elaborate experimental structure than we have achieved in the present series. Nevertheless, the analyses have allowed the assessment of dominance and interaction in the data so that $\mathrm{C}$ can be interpreted accordingly.

In the 1953 experiment, where the analyses reveal simple additivity and no dominance for the genes concerned, we can assume that $\mathrm{C}$ depends entirely on reinforcing hom/hom combinations $\left(D^{\prime}\right)$, and continued perfect or strong assortative mating should fairly rapidly achieve fixation of genetic variability. But as Wright (l.c.) points out, the variability would be fixed for the most part in the free state as multiple reinforcement homozygotes and not as for selfing, where, in the absence of linkage equal proportions of free and homozygous potential variation (opposition homozygotes) are obtained. Furthermore, fixation will be achieved only within the assorted character, other characters, as is seen from a consideration of the height data, being affected only to the extent that they are correlated in expression with the assorted character. In the $195^{2}$ experiment where $\mathrm{C}$ for flowering time is also dependent on hom/het and het/het combinations ( $\mathrm{B}, \mathrm{B}^{\prime}$ and $\mathrm{H}^{\prime}$ items assuming only dominance), the advance to the fixation of free variability must still continue though at a slower rate. If we assume the genetic control to be more complex-involving genic interaction and linkage (especially in repulsion)-the fixation process is likely to be still further retarded but cannot be halted if the degree of genotypic correlation achieved by continued phenotypic assortment is sufficiently high.

Assorting on flowering time in the $195^{2}$ experiment also achieved a significant association of plants alike in height, and in the resultant generation it was possible to demonstrate significant $\mathrm{C}$ for this character. This effect may be deemed analogous to the correlated response 
observed by Mather and Harrison (1948) as decreased fertility arising from selection for high and low chæta number in Drosophila melanogaster. That no such effect was observed in the 1953 experiment can, of course, be explained by the large sampling variation attendant upon the low flowering time/height correlation $(r \bumpeq 0.45$ in both experiments). The r952 results also illustrate that where genetic control is uncomplicated by dominance or interaction, a comparatively low order of phenotypic correlation (in this case $r=0.38$ ) is sufficient to evoke an observable response with regard to the distribution of heritable variation in succeeding generations.

The fact that we have been able to demonstrate significant changes in the distribution of heritable variation, although considering but a single generation, suggests that assortative mating may profitably have wider use as a technique in plant or animal breeding, especially where inbreeding methods are impracticable or undesirable. In experimental populations error variation tends largely to be under control so that a high degree of genotypic correlation should be attainable by perfect assortative mating. Although initially assortative mating does little to upset the homozygotic/heterozygotic balance, the heterozygotic state is maintained chiefly at the expense of the balanced or opposing homozygotic combinations, the free variability becoming gradually fixed within the population. Thus, since the breeder is usually concerned with extreme expression, selection based on assortative mating is not only useful as a practical means of securing the appearances which he requires (Nichols, 1947), but if continued should achieve the fixation of desired phenotypes without recourse to inbreeding. Moreover, where the genes controlling the character tend to be linked in opposition, this method of selection, by avoiding the premature homozygosis and fixation resulting from any system of inbreeding, should maximise the chances of securing and ultimately fixing desirable recombinants.

This could prove of particular value in the treatment of species showing marked inbreeding depression. Jinks (r954) has put forward the view that apparent overdominance appearing in maize and other species can be related largely to interaction between non-allelic genes. The rapid loss of vigour on inbreeding the hybrids can then be explained as close linkage in opposition of genes which show complete dominance and genic interaction. Should assortative mating be initiated among such hybrids and their derivatives, the frequencies of gene combinations giving extreme expressions would be maximised in certain of the resultant families. If the linkage relationships of the genes are such that favourable expression depends on heterozygotic (relational) balance, heterozygosis must initially be retained, but again the chances of procuring and retaining desirable recombinants would be maximised at each generation. Continued selection based on assortative mating should then increase the frequencies of such recombinants until they ultimately become fixed in the homozygous state. Furthermore, we 
have seen that the extent to which other characters are affected depends on the strength of their correlation with the assorted character. Provided this correlation is not complete, it follows that this method of selection can also allow reassociation of genes controlling different characters. Thus favourable expression in characters other than that upon which selection is primarily based can be maintained or finally achieved.

Evidence deriving from recent selection experiments is manifestly in agreement with these views. For instance, the experiments of Mather and Harrison (1949) involved selection based on the assortative mating of extreme phenotypes in such a manner that inbreeding was reduced to a minimum. Although initially response to selection for high and low chæta number was accompanied by decreased fertility, they ultimately established true breeding lines having restored fertility and with chæta numbers considerably outside the range of variation observed in the original population. Admittedly, where the genetical situation is complicated, progress towards the fixation of desirable phenotypes is likely to be slow and improvement based on assortative mating would require long-term breeding programmes. In this respect further information as to the effect of assortative mating on a character showing high genic interaction may prove informative to breeders in general and those dealing with outbreeding crops in particular. Coupled with experiments designed to test the effects of assortative mating when combined with varying degrees of inbreeding in later generations, this information may reveal methods of dealing with material showing marked inbreeding depression in such a way that ultimately the commercial breeder need no longer depend upon the constant production of hybrids in the attainment of "hybrid vigour".

Where the genotypic correlation achieved between parents is low, either by virtue of low phenotypic correlation (partial assortative mating) or because of high environmental variation, the degree of fixation will be smaller and the process being relatively slow will achieve importance only when measured on an evolutionary time scale. That a degree of assortative mating does occur as a natural phenomenon is evident, since before interbreeding can occur with any freedom prospective mates must occupy the same ecological niche and have their mating seasons coincidental. For instance, where a crossbreeding species exhibits variation in flowering time, interbreeding will be more frequent between plants flowering at the same time.

In a randomly breeding population the relative proportions of free and homozygous potential variation depends on the linkage relationships of the different genes. We have seen that where assortative mating is operative the proportion of free variability tends to be increased for the character in question at the expense of the homozygous potential variability. Populations affected must therefore 
eventually reach a new equilibrium depending on the degree of genotypic correlation achieved by the mating system, such that with complete genotypic correlation the variability becomes finally fixed in the free state (see Wright, l.c.). Thus assortative mating can effect divergence within the population and if sufficiently strong can of itself form a basis for speciation. Furthermore, in crossbreeding species, the linked combinations of genes are likely to be relationally rather than internally balanced (Darlington and Mather, 1949), and " inbreeding depression" follows any departure from a crossbreeding system; but if we again explain relational balance as close linkage in opposition of genes showing interaction and/or dominance, assortative mating increases the frequency with which such linked combinations are broken and liberated to the action of selection as novel homozygotic combinations. In this way internal balance may be built up, and once established, would allow the development of inbreeding systems in species hitherto only able to survive as outbreeding units.

From the evolutionary standpoint, therefore, assortative mating can be both a force and a process. As a force it can of itself provide the impetus for divergence and speciation; as a process it provides a means whereby internal chromosomal balance may become established, and thus the first step to facultative inbreeding achieved, in hitherto obligatory outbreeding species.

\section{SUMMARY}

I. The possible consequences of a system of assortative (i.e. like to like) mating in quantitative inheritance have been considered for situations involving differing conditions of dominance. Experiments in Nicotiana rustica designed to compare the effects of assortative $(A)$ relative to selfing $(F)$ and random $(R)$ mating systems have been analysed by means of the biometrical method of analysis developed by Mather (1949a and $b$ ), using flowering times as the continuously varying character.

2. The experiments demonstrate a significant increase in the genetic variability of $A$ family means compared with that found under random mating. The extent of this increase depends (i) on the phenotypic correlation achieved between parents; (ii) on the degree to which genic expression is affected by environmental fluctuations; and (iii) on the complexity of the genetic control of the character. In no case was the genetic variation of $A$ progenies so great as that found in the $F$ generation.

3. If the numbers of genes involved is large, the increased variance of the $A$ families cannot initially be attributed to any change in the homozygote/heterozygote ratio relative to that found under random mating. It depends, rather, on a re-association of the genes in such a way that an excess of combinations giving extreme expression is achieved at the expense of the more balanced combinations. Where 
dominance is absent this must rapidly result in the homozygosis of reinforcing genic combinations and hence fixation of extreme expressions. With more complex genetic situations involving dominance, genic interaction and linkage, this process may be retarded but not necessarily averted.

4. The possible use of assortative mating as a technique in plant or animal breeding has been discussed. In evolution assortative mating is regarded as a force in the splitting of populations and hence speciation. It is also a process whereby the achievement of internal chromosomal balance may be accomplished. This is the initial and most important step towards the establishment of facultative inbreeding in hitherto obligate outbreeding species.

Acknowledgments.-I am indebted to Professor K. Mather, Dr B. I. Hayman and Dr J. L. Jinks for helpful discussion throughout the course of the work described in this paper.

\section{REFERENCES}

Darlington, C. D., AND MATHER, K. 1949. The Elements of Genetics. Allen and Unwin, London.

HAYMAN, B. 1. 1953. Components of variation under sib-mating. Heredity, 7, $121-125$.

hayman, B. I. 1954. Ph.D. Thesis, University of Birmingham.

JiNKS, J. L. 1955. A survey of the genetical basis of heterosis in a variety of diallel crosses. Heredity, 9, 223-238.

MATHER, K. 1949a. Biometrical Genetics. Methuen, London.

MATHER, K. 1949b. The genetical theory of continuous variation. Hereditas, Suppl. Vol.

MATHER, K., AND BATEMAN, A. J. 1951. The progress of inbreeding in barley. Heredity, 5, 321-348.

MATHER, K., AND HARRison, B. J. 1949. The manifold effect of selection. Heredity, $3,1-52$ and $151-162$.

MATHER, K., AND viNES, A. 1952. The inheritance of height and flowering time in a cross of Nicotiana rustica. Quantitative Inheritance. H.M.S.O., pp. 49-8o. Nichols, J. E. 1947. Livestock Improvement. Oliver and Boyd, Edinburgh. WRIGHT, S. 1921. Systems of mating, I-IV. Genetics, 6, 111-178. 